\title{
Freier Zugang zur juristischen Fachliteratur im Spiegel der Open-Science-Bewegung
}

Reflexionen zur Tagung «Open Access in den Rechtswissenschaften» vom 27. Mai 2016 an der Universität Bern

Hanjo Hamann *

Die Wissenschaftskommunikation befindet sich weltweit im Umbruch: Einflussreiche Forschungsinstitutionen wollen bis 2020 die Resultate aller öffentlich finanzierten Forschung frei im Internet zugänglich machen. Die sogenannte Open-AccessBewegung wird auch die Rechtswissenschaft in ihren Grundfesten erschüttern, wenn Juristen nicht frühzeitig vorbauen. Nur eine breite und ergebnisoffene Diskussion über die Ursachen, Potentiale und Gefahren von Open Access kann verhindern, dass die Besonderheiten der juristischen Fachkultur und die gewachsenen Strukturen ihrer Verlage in den entscheidenden Debatten übersehen oder übergangen werden. Diese Diskussion wurde nun durch eine Tagung an der Universität Bern eröffnet, die neue Impulse gesetzt hat und zu weitergehenden Reflexionen über den Mehrwert von Open Access, den bisherigen Widerstand vieler Rechtswissenschaftler und die künftige Rolle der juristischen Fachverlage anregt.

I. Einleitung. .97

II. Perspektiven auf juristisches Open Access …………........................................... 97

1. Theorie .................................................................................................. 97

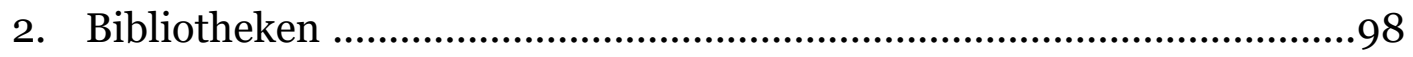

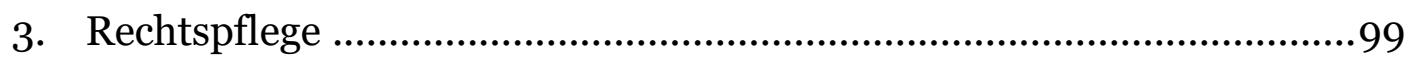

4. Infrastrukturanbieter ……………….................................................100

5. Wissenschaftsnachwuchs ...................................................................100

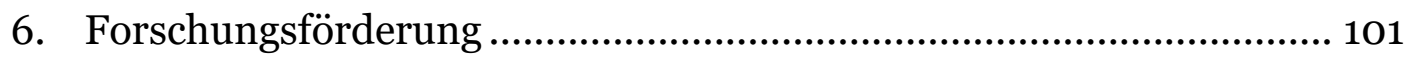

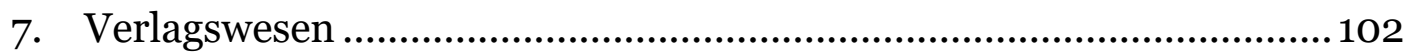

III. Offene Flanken des juristischen Open Access................................................. 102

1. Wozu Open Access in den Rechtswissenschaften? ..................................103

2. Woher der Widerstand von Juristen gegen Open Access? .................... 103

3. Welche Rolle spielen juristische Verlage für Open Access? ..................104

IV. Zukunftsperspektiven und Weiterführung ..........................................................104

Zitiervorschlag: Hanjo Hamann, Freier Zugang zur juristischen Fachliteratur im Spiegel der Open-Science-Bewegung, in: sui-generis 2016, S. 96

URL: $\quad$ sui-generis.ch/28

DOI: $\quad$ https://doi.org/10.21257/sg.28

* Dr. iur. Dr. rer. pol., wissenschaftlicher Mitarbeiter und Open Access Ambassador, Max-PlanckInstitut zur Erforschung von Gemeinschaftsgütern (Bonn), Fellow Freies Wissen der WikimediaStiftung, Managing Editor der Open-Access-Zeitschrift «Language \& Law» (JLL).

Dieses Werk ist lizenziert unter einer Creative Commons Namensnennung - Weitergabe unter gleichen Bedingungen 4.0 International Lizenz. 


\section{Einleitung}

1 Im internationalen Wissenschaftsdiskurs formiert sich derzeit eine Bewegung für offene Wissenschaftspraktiken, die auch den freien Zugang zu öffentlich finanzierter Forschung durch (Rück-)Vergemeinschaftung der Forschungsliteratur anstrebt. ${ }^{1}$ Jedermann, so ihr Plädoyer, solle über das Internet auf alle Forschungsergebnisse ohne Beschränkungen offen zugreifen können - auf Englisch: Open Access $(\mathrm{OA})$.

2 Waren Open-Access-Veröffentlichungen bislang vor allem Gegenstand der juristischen Forschung, ${ }^{2}$ erreichten sie die deutschsprachige Rechtswissenschaft am 27. Mai 2016 auch als binnendisziplinäre Methodenfrage. 3 Darüber zu diskutieren hatten Wissenschaftskommunikator Dirk Verdicchio und Rechtslehrer Daniel Hürlimann mit Unterstützung der Bibliothekare Bernhard Dengg und Regula

Zum Hintergrund Hamann, RW 2016, 323.

Monographisch aus Deutschland etwa Spindler (Hrsg.), Rechtliche Rahmenbedingungen von Open Access-Publikationen 2006; Hirschfelder, Anforderungen an eine rechtliche Verankerung des Open Access Prinzips 2008; Krujatz, Open Access. Der offene Zugang zu wissenschaftlichen Informationen pp. 2012; Lutz, Zugang zu wissenschaftlichen Informationen in der digitalen Welt 2012; Lucyga, Open Access - Möglichkeiten und Grenzen 2015; Schmidt, Open Access. Hochschulrechtliche Veröffentlichungs- und urheberrechtliche Anbietungspflichten des Hochschulprofessors 2016; in Aufsatzform bspw. Hirschfelder, JurPC Web-Dok. 46/2009; Sosnitza, RW 2010, 225; Peukert, JIPITEC 2012, 142; Bäuerle, in: Britz (Hrsg.), Forschung in Freiheit und Risiko 2012, S. 1 ff.; Peukert, in: Grünberger/Leible (Hrsg.), Die Kollision von Urheberrecht und Nutzerverhalten 2014, S. 145 ff.; Krausnick, in: Geis u.a. (Hrsg.), Von der Kultur der Verfassung - FS Hufen 2015, S. 367 ff.; Fehling, in: HoffmannRiem (Hrsg.), Innovationen im Recht 2016, S. $337 \mathrm{ff}$.

3 Dazu bisher etwa Abegg, Jusletter 1.9.2008; Zimmermann, NJW 2014, 3000 f.; Herb, JurPC WebDok. 5/2015; Hamann, RW 2016, 323; Hamann, blogdroiteuropéen 25.10.2016.
Schatzmann an die Universität Bern eingeladen. Dieser Einladung folgten ein deutscher und fünf Schweizer Referenten nebst über 50 Teilnehmern, davon drei aus Deutschland. Die Teilnehmerliste weist einen vielfältigen Teilnehmerkreis aus: Von 58 Angemeldeten (inkl. Referenten) kamen $36 \%$ aus dem Bibliothekswesen, $17 \%$ aus der Wissenschaft und $12 \%$ aus Verlagen, die restlichen verteilten sich gleichmässig auf Rechtspflege, Unternehmen, Politik und Verwaltung.

3 Der Vielfalt des Publikums entsprach die Vielfalt der Perspektiven auf das Tagungsthema: Nach kurzem Grusswort des Berner Bibliotheksdirektors Niklaus Landolt beleuchteten sechs zwanzigminütige Vorträge mit je zehnminütiger Aussprache das Spannungsfeld von Open Access in den Rechtswissenschaften. Diese vielfältigen Blickwinkel der Tagung (dazu II.) führen auf wichtige Grundfragen (III.) und Zukunftsimpulse (IV.).

\section{Perspektiven auf juristisches Open Access}

4 Es genügen jeweils kurze Zusammenfassungen der Vorträge: Deren Folien wurden im gelungenen Einklang mit dem Tagungsthema im Berner Universitätsrepositorium frei zugänglich gemacht.

\section{Theorie}

5 Im Eröffnungsvortrag stellte Dirk Verdicchio (Leiter der Fachstelle Open Access und Wissenschaftskommunikation der UB Bern) fest, «dass Open Access in den Rechtswissenschaften relativ wenig verbreitet ist und kaum diskutiert wird", und führte deshalb in «aktuelle Entwicklungen im Bereich des Open Access» ein: 
Open Access als Veröffentlichungsform sei nach Peter Suber dadurch gekennzeichnet, dass sie erstens «digital, online und kostenfrei» erfolge und zweitens «frei von den meisten Urheberrechtsund Lizenzbeschränkungen». Davon abzugrenzen sei die blosse Verfügbarmachung («Free Access»), ohne dass diese Abgrenzung im Weiteren noch einmal aufgegriffen worden wäre. Stattdessen stellte der Referent empirische Erhebungen vor - besonders bezeichnend wohl eine Umfrage unter gut $38^{6} 000$ Wissenschaftlern im Jahr 2011, der zufolge Open Access unter Juristen mit 90 \% eine Zustimmungsquote im Mittelfeld aller Disziplinen verzeichnete, noch vor Psychologie, Physik und Chemie.4 Danach erläuterte er die Open-Access-Aktivitäten der EU, Skandinaviens, der Niederlande, der angelsächsischen und deutschsprachigen Länder und hob in Deutschland vor allem jene von DFG und Max Planck Digital Library (MPDL) hervor.

\section{Bibliotheken}

6 Im anschliessenden Überblick über «bestehende Infrastrukturen» und den «derzeitigen Publikationsmarkt» spekulierte Bernhard Dengg (Leiter der juristischen Bibliothek der UB Bern), dass sich Open Access in den Rechtswissenschaften aus drei Gründen nicht durchsetzen werde: Wegen gewachsener Publikationsstrukturen, der juristischen Wissenschaftskultur und eines Mangels an Anreizen. Insbesondere das im deutschen $\S 38$ IV 1 UrhG vorgesehene und in der Schweiz umstrittene Zweitveröffentlichungsrecht als «Spitze der Lanze der Open-Access-

Dabei, so wäre zu ergänzen, stellten Juristen aber from the SOAP project survey, S. 3).
Bewegung» werde stumpf bleiben. Zum Beleg dafür diente eine eigene Erhebung, nach der von 9'333 Fachaufsätzen, die in 6o Schweizer Rechtszeitschriften 20102015 erschienen waren, zwar $45 \%$ von Juristen im Wissenschaftsbetrieb stammten, davon aber lediglich $18 \%$ online abrufbar waren: Trotz der kurzen und grosszügig gehandhabten Embargofristen der wichtigsten Schweizer Verlage Dike, Schulthess und Stämpfli (je 3-6 Monate) seien von keinem Verlag mehr als 28 \% der Aufsätze auf der Website ihres jeweiligen Autors auffindbar. Das lasse sich durch die überschaubaren Abonnementkosten in der Schweiz erklären: Alle Schweizer Rechtszeitschriften kosteten geschätzt nur CHF 600'0oo, deshalb zeigte der Vortrag unter der vielsagenden Folienüberschrift «Open Access? - No Chance!» auf, dass «niemand Interesse daran [habe], etwas zu ändern», solange Open-Access-Zeitschriften den Printmarkt «nur imitieren», denn: «Geld regiert die Welt». Bedauerlich sei das insoweit, als eine «elektronische Auswertung von Textmengen» nicht möglich sei: «Wie soll ich jemals eine Text und Data Mining Untersuchung über die Datenbanken laufen lassen?»

7 Dieser Vortrag war sogleich der kontroverseste des Tages: Aus sechs Wortmeldungen sind vor allem die von Prof. Thomas Koller (Universität Bern) und Beat Immenhauser (Schweizerische Akademie der Geistes- und Sozialwissenschaften) hervorzuheben, die den Schluss von bisherigen Gepflogenheiten auf deren Unabänderlichkeit für verkürzt bzw. «zirkulär» hielten. Koller verglich den früher als Open-Access-Zeitschrift erschienenen «Jusletter», der Aufsätze bis zu 80 Seiten innerhalb von zwei bis acht 
Wochen publiziere, mit deutschen Printzeitschriften, die dafür mitunter acht bis zehn Monate brauchen: Die schnellere Veröffentlichung (und grössere Sichtbarkeit bei 20'00o Newsletter-Empfängern) schaffe durchaus Anreize zur OpenAccess-Veröffentlichung. Ingrid Kissling (s.u. 6.) wies darauf hin, dass frei zugängliche Veröffentlichungen nur ein Baustein der Open-Science-Bewegung seien, die eine allgemeinere Reflexion über Wissenschaftsstandards und Evaluationsmethoden anstrebe: «Gehen diese Diskussionen an den Jurist/innen vorbei?» Eine Antwort hierauf blieb die Tagung schuldig. 5

\section{Rechtspflege}

8 Urheberrechtliche Aspekte beleuchtete anschliessend RA Matthias Seemann (Zürich), der den «Zugang zu wissenschaftlichen Publikationen im schweizerischen Recht» gemeinsam mit Reto Hilty 2009 ausführlich begutachtet hatte. Nach allgemeiner Erläuterung des Schweizer Urheberrechts, das anders als in Deutschland übertragbar sei, erörterte er sowohl das «Recht auf Open-AccessVeröffentlichung» (Art. 382 OR sehe ein gesetzliches Verlagsvertragsrecht vor: grundsätzlich exklusiv, aber für Zeitschriftenbeiträge nur dreimonatige Karenz) als auch «Pflichten zur OpenAccess-Veröffentlichung»: Diese können einerseits aus Förderrichtlinien folgen (so grundsätzlich bei Projekten mit nationaler Forschungsförderung), andererseits aus dem Hochschulrecht, wobei die Open Access Policies der Schweizer

\footnotetext{
Die deutsche juristische Aufsatzdatenbank Juris verzeichnet keinen einzigen Literaturnachweis für «Open Science»; Nachweise zu Evaluationsmetriken finden sich immerhin bei Hamann, RW 2014, 501, $506 \mathrm{f}$.
}

Hochschulen aber nur «eine gewisse Erwartung» ausdrückten und im Übrigen die Freiheit der Publikationswahl betonten.

9 Sodann kam eine aktuell vorgesehene Revision des Schweizer Urheberrechts zur Sprache, deren Vernehmlassung Ende März 2016 diverse Vorschläge zur Ermöglichung von Open Access ergeben habe. (Rückfrage nach dem Vortrag ergab, dass hierauf noch keine Reaktion absehbar sei, da mit gut 1'30o Zuschriften zehnmal so viele eingegangen seien wie zur letzten Urheberrechtsrevision womöglich folge deshalb gar noch eine zweite Vernehmlassung.) Abschliessend erörterte der Vortrag drei rechtstechnische Möglichkeiten, Open Access zu fördern: Durch Urheberrechtsschranke wie in England ( $§ 29 \mathrm{~A} \mathrm{CDPA}$ ), Zweitveröffentlichungsrecht wie in Deutschland (§ 38 IV 1 UrhG) oder kartellrechtliche Preiskontrolle - etwa gegenüber Verlagskonzernen wie Elsevier und Springer. Jede rechtliche Gestaltung stehe jedoch im Spannungsfeld der Interessen, das zumindest die Publikationsfreiheit von Autoren, die Vertragsfreiheit von Verlagen, das Verbreitungsinteresse von Repositorien (und, so wäre zu ergänzen, Bibliotheken) und das Interesse von Universitäten an der Förderung ihrer Forschung umfasse.

In der anschliessenden Aussprache ergänzte Beat Immenhauser die offene Frage, wie Autoren ihr Zweitveröffentlichungsrecht gegen internationale Verlage durchsetzen könnten. Der Referent verwies hierzu auf eine Vernehmlassungsantwort von vier Rechtsprofessoren, wonach Rechtswahlklauseln im Verlagsvertrag unwirksam sein sollten. 


\section{Infrastrukturanbieter}

11 Nach der Pause eröffnete Ivo Vogel (Leiter des Fachinformationsdienstes für internationale und interdisziplinäre Rechtsforschung der Staatsbibliothek zu Berlin) die zweite Hälfte der Tagung mit Überlegungen zum «Open Access für die deutsche Rechtswissenschaft». Er erläuterte zunächst, dass umstritten sei, ob etwaige Pflichten zur Open-Access-Veröffentlichung in das (durch Art. 5 III GG geschützte) Recht von Wissenschaftlern eingreifen, über Zeitpunkt und Umfang der Veröffentlichung zu entscheiden, oder ob dadurch lediglich die Wahl des Veröffentlichungsmediums (und gerade nicht das Forschungsgrundrecht) beschränkt werde. Er illustrierte dies an $\S 44 \mathrm{VI}$ des baden-württembergischen Hochschulgesetzes, der Hochschulen dazu anhält, ihre Wissenschaftler durch Satzung zur Ausübung ihrer Zweitveröffentlichungsrechte zu verpflichten: Gegen die Umsetzung in $\S 2$ der Konstanzer Universitätssatzung habe sich ausgerechnet die juristische Fakultät verwehrt. Der Ausgang des Streits sei wegweisend für die weitere Entwicklung - und angesichts der hochschulrechtlichen Publikationspflicht völlig offen (§ 2 V 2 LHG BW: «Hochschulen [...] fördern [...] den freien Zugang zu wissenschaftlichen Informationen»).

Danach stellte der Vortrag die deutschen Infrastrukturen dar: Führend in der Open-Access-Förderung sei die MaxPlanck-Gesellschaft (MPG); ihre bislang einzigartige Schriftenreihe "Global Perspectives on Legal History» sei «pures Open-Access-Gold». Die wegweisende Springer-Compact-Vereinbarung der MPG betreffe jedoch nur drei randständige Rechtszeitschriften, und auch das Publikations-Repositorium «PuRe» verzeich- ne kaum Volltexte aus den zehn juristischen Instituten - das Strafrechtsinstitut fehle sogar völlig.

13 Daneben sei die DFG der «zweite grosse Player für die Open-Access-Aktivitäten in Deutschland». Deren Publikationsfonds dienten allerdings nur zur Anstossfinanzierung und liefen oft aus, weil Universitäten die Anschlussfinanzierung vernachlässigten. Im Projekt «DeepGreen» wandle die DFG derzeit Texte in Open Access um, die aufgrund überregional verhandelter «Allianz-Lizenzen» schon jetzt frei zugänglich gemacht werden dürften.

14 Ansonsten sei die Bilanz mager: In der Bielefelder Suchmaschine für OpenAccess-Dokumente verzeichne «Recht» unter allen sozialwissenschaftlichen Disziplinen die drittwenigsten Suchtreffer gerade noch vor «Bräuche, Etikette, Folklore» und «Öffentliche Verwaltung, Militärwissenschaft». Im Verzeichnis der Open-Access-Zeitschriften seien nur zwei in Deutschland herausgegebene Rechtszeitschriften verzeichnet (GoJIL und Ancilla Iuris), in der entsprechenden Buchdatenbank nur 236 juristische Titel. Einen «goldenen Lichtstreif» bildeten lediglich die Wissenschaftsblogs (namentlich Humboldt Law Clinic, Völkerrechts-, Verfassungs- und JuWissBlog), denen der Fachinformationsdienst eine Langzeitarchivierung anbiete.

\section{Wissenschaftsnachwuchs}

15 Im vorletzten Vortrag über «Open Access als Karriereinstrument» berichtete $D a$ niel Hürlimann (Ass.-Prof. für Informationsrecht der Universität St. Gallen) über seine Gründung der Open-AccessZeitschrift sui-generis.ch im Jahr 2014: Der zunächst um ideelle Unterstützung 
angegangene Dekan einer Rechtsfakultät habe erwidert, «offener Zugang» sei für ihn die Möglichkeit, Zeitschriften in der Bibliothek einzusehen; Open Access setze dagegen PC und Internet voraus - «das habe nicht jeder».

Die gleichwohl gegründete Open-AccessZeitschrift habe sich bewährt: Ihr Verzicht auf feste, termingerecht zu füllende Heftstrukturen ermögliche höhere Qualitätsanforderungen; auch die Öffentlichkeitswahrnehmung steige spürbar, wenn Journalisten keinen Datenbankzugang erwerben oder sich Passworte merken müssen, wie es der Referent in jahrelanger Pressearbeit für einen kommerziellen Verlag erlebt habe. Den sprichwörtlichen «Open-Access-Zitationsvorteil» belege jüngst der Fall einer Nachwuchsautorin, die aufgrund ihres Beitrags in der Zeitschrift als Expertin in eine Fernsehsendung eingeladen worden sei. Auch für ihn selbst habe sich das Open-AccessEngagement ausgezahlt und nachweislich seine Berufung nach St. Gallen befördert.

Auf Nachfrage ergänzte er, dass auch die übrigen Rückmeldungen auf seine Zeitschrift durchweg positiv gewesen seien: Von jeder Schweizer Universität habe sich ein Mitstreiter für die achtköpfige Redaktion finden lassen, der Name der Zeitschrift stamme gar von einem Zürcher Ordinarius. In einer weiteren Wortmeldung ermutigte Thomas Koller zur aktiveren Werbung für die Zeitschrift, die bislang noch zu wenig bekannt sei.

\section{Forschungsförderung}

18 Im letzten Vortrag des Tages präsentierte Ingrid Kissling (Leiterin der Abteilung Geistes- und Sozialwissenschaften beim
Schweizerischen Nationalfonds) die Sicht der Forschungsförderer auf «Open Access auch in den Rechtswissenschaften». Sie betonte noch einmal die nach dem ersten Vortrag angemahnte Einbettung von Open Access in die Open-ScienceBewegung und verwies auf den «Amsterdam Call for Action», der bis 2020 alle öffentlich finanzierte Forschung auf Open-Access-Veröffentlichung umstellen wolle. Dies sei eine «starke Bewegung, die schnell voranschreitet und auch gepusht wird». Das zeigten nicht zuletzt sogenannte «Offsetting-Agreements», durch die Forschungseinrichtungen einzelnen Verlagen die Übernahme von Veröffentlichungskosten für Autoren dieser Einrichtungen zusagen; davon seien in den Niederlanden schon fünf bekannt, in Österreich zwei, in Deutschland einzig die Springer-Compact-Vereinbarung der MPG, in der Schweiz bislang keine. Doch erwarteten nationale Forschungsförderer auch hier, dass «mit öffentlichen Geldern erarbeitete Forschungsresultate weltweit kostenlos zugänglich» würden. Deshalb müssten Zeitschriftenbeiträge sofort Open Access veröffentlicht und Bücher spätestens nach einer Embargofrist online gestellt werden («Gold Road Journals, Green Road Books»). Auf Nachfrage räumte sie freilich ein, dass Verstösse gegen diese Richtlinien bislang grosszügig gehandhabt würden - was ihre für die Rechtswissenschaft zuständige Kollegin Beatrice Tobler bestätigte («von Sanktionen sind wir noch weit entfernt»), dabei aber zugleich die Möglichkeit von Sanktionen betonte.

19 Im zweiten Teil ihres Vortrags stellte die Referentin die SNF-Studie «OAPEN» vor, deren Auswertung momentan läuft: In einer ersten Ausschreibung habe der 
SNF 56 Bücher teils als Print-, teils als Open-Access-Veröffentlichungen gefördert, um vergleichend zu erfahren, was ein Buch koste, was Open Access für den Absatz bedeute, wie oft solche Bücher heruntergeladen würden, ob dadurch hohe Einbussen entstünden oder beide Veröffentlichungsarten komplementär nebeneinander bestehen könnten, etc. Die zweite Ausschreibung (die erstmals auch juristische Monographien umfassen wird) laufe derzeit, und auch im Bereich der Projekt-Berichterstattung lote der SNF im neuesten Monitoringbericht Verbesserungspotential aus: So werde bislang etwa die Hälfte der Open-AccessVeröffentlichungen aus SNF-Projekten gar nicht gemeldet, aber auch der berichtigte Anteil betrage in der Rechtswissenschaft nur $16 \%$ - lediglich Theologie/ Geschichte liege noch darunter, während Naturwissenschaften bis zu 80 \% der SNF-geförderten Arbeiten im Open Access veröffentlichten.

Zuletzt stellte die Referentin die Anregungen des SNF zur bevorstehenden Urheberrechtsrevision (Zweitveröffentlichungsrecht, Urheberrechtsschranke, Verkürzung der Urheberschutzfrist) und ein laufendes Projekt zur Aufklärung der Finanzflüsse im Veröffentlichungswesen vor. Hierzu sollen bis Herbst Zahlen vorliegen ${ }^{6}$, um den Rückstand der Schweiz als «Forschungs- und Bildungsnation» aufzuholen. Mit diesen schicksalsschwangeren Worten endete der letzte Vortrag der Tagung.

Der Bericht «Financial Flows in Swiss Publishing - Final Report» wurde am 18. November 2016 publiziert.

\section{Verlagswesen}

21 Die siebte der sechs Perspektiven der Tagung fehlte, obwohl ihre Vertreter im Raum sassen: Kein Verlag war im Vortragsprogramm vertreten - was weniger den Organisatoren anzulasten sein dürfte, die dem Vernehmen nach frühzeitig mit Verlagen in Kontakt standen. Doch lässt sich die für eine zielführende Entwicklung von Open Access unverzichtbare Perspektive der Verleger7 wenigstens aus informellen Randbemerkungen rekonstruieren: Im Pausengespräch meinte beispielsweise der Geschäftsführer eines namhaften Schweizer Fachverlags, die Open-Access-Debatte verkürze das Publikationswesen darauf, Träger von Information zu sein. Zu kurz komme die sinnliche Peripherie als eigentlicher Mehrwert der Verlage: Haptik des Druckwerks, aufwändige Vermarktung, Emotionalisierung des Buchprodukts, usw. Dennoch hielten sich die im Publikum fast vollzählig vertretenen Schweizer Rechtsverlage in der Diskussion durchweg zurück. Allein Hermann Rucktäschel vom Stuttgarter Boorberg-Verlag erläuterte nach dem vierten Vortrag, Verlage suchten noch tragfähig finanzierbare Open-Access-Geschäftsmodelle, etwa in Form von «Inhaltsveredelung» und selektiver Sichtbarmachung, denn Repositorien seien letztlich «x-beliebig» - «damit fängt der Jurist nichts an».

\section{Offene Flanken des juristischen Open Access}

22 Aus dem Gesamtbild der Tagung ergeben sich drei übergreifende Fragestellungen für eine breitere Diskussion und Vertiefung:

7 Dazu Hamann, RW 2016, 323, 328. 


\section{Wozu Open Access in den Rechts- wissenschaften?}

23 Diese Frage durchzog alle Vorträge, die Antwort wurde aber eher unterstellt denn expliziert. $\mathrm{Zu}$ bekannt sind wohl mittlerweile die Argumente der OpenAccess-Befürworter für den freien $\mathrm{Zu}-$ gang zu öffentlich finanzierter Forschung. Darüber hinaus kam vor allem die bessere Auswertbarkeit grosser Textmengen zur Sprache: Ein Rückfrager zum zweiten Vortrag zweifelte zwar, ob Juristen überhaupt an computergestützter Massentextauswertung (sogenanntem Textmining) interessiert seien, woraufhin der Referent auf die Erleichterung des für Bibliothekare entscheidenden bibliographischen Überblicks verwies. Zu ergänzen wäre noch, dass auch die interdisziplinäre Rechtsforschung von Textmining profitieren würde: Unter dem Stichwort «Rechts(korpus)linguistik» befassen sich z.B. das Zürcher Zentrum für Rechtsetzungslehre und das deutsche Akademieprojekt «Computer Assisted Legal Linguistics» mit der computergestützten Massentextauswertung. Ähnliches gilt für die stärker informatisch motivierten Zugänge des sogenannten «Legal Tech» (z.B. lexalyze.de). Sie alle benötigen als Rohmaterial juristische Fachtexte in fehlerbereinigten elektronischen Formaten, deren Herstellung im Printzeitalter noch enormen Zeit- und Geldaufwand bereitet.

\section{Woher der Widerstand von Juris- ten gegen Open Access?}

24 Diese Frage schien viele Tagungsteilnehmer zu bewegen, wurde aber nur teilweise beantwortet, bevor Christian Schlumpf (Fachreferent der Universitätsbibliothek St. Gallen) sie nach dem vierten Referat aussprach und Ivo Vogel ein Generationenproblem vermutete, das aber die Frage offenlasse: «In anderen Disziplinen klappt's, warum nicht auch bei den Juristen?» Der hiesige Autor wies dazu auf drei Probleme hin: Erstens sei vielen Juristen das Konzept «Open Access» schlicht nicht vertraut - selbst die gar nicht mehr so seltenen Beispiele juristischen Open-Access-Engagements (wie die Zeitschriften Ancilla Iuris, BLJ, FoR, FHI, FreiLaw, GLJ, GoJIL, HFR, HRRS, JIPITEC, JLL, JSE, JurPC, MIR, OdW, Rg, sui generis, ZIS, ZJS oder die Monographie von Schweizer 2005) werden fast nie mit diesem Etikett versehen und scheinen sich der möglichen Eingliederung in etablierte Open-Access-Strukturen (z.B. DOAJ, s.o. I.4) gar nicht bewusst. Zweitens seien einzelne Wissenschaftler durchaus widerwillig, wie der fünfte Vortrag illustrierte - zumal die Rechtswissenschaft (zumindest in Deutschland) eine der letzten Disziplinen sein dürfte, in der Wissenschaftler ihre Aufsätze noch vergütet bekommen und deshalb finanzielle Eigeninteressen am tradierten Veröffentlichungswesen haben. Drittens sei der Verlagsmarkt überschaubar und massgeblich durch KMU geprägt, die sich Experimente kaum leisten können und in einem von konservativen Erwartungen geprägten Umfeld nicht den ersten Schritt wagen wollen. Dazu tritt wohl, viertens, die institutionelle Besonderheit der Rechtswissenschaft, dass - folgt man der Statistik aus dem zweiten Vortrag - nur die Hälfte der Fachtexte von Wissenschaftlern stammen. Die übrigen Autoren aus Rechtspraxis und Wirtschaft würden mittelbar subventioniert, wenn Fachzeitschriften vom Abonnementverkauf auf öffentliche Direktfinanzierung umstellten. Abhilfe liesse sich 
vielleicht durch mischfinanzierte Modelle oder Fallstudien zu den von Rechtspraktikern begründeten Open-Access-Zeitschriften (etwa HRRS und MIR) entwickeln.

\section{Welche Rolle spielen juristische Verlage für Open Access?}

Ein Auslöser für die Open-Access-Bewegung in den Naturwissenschaften war das Gefühl, Wissenschaftsverlage seien befördert durch Konzernierung und Aktionärserwartungen - aus der Rolle des Mehrwert-Dienstleisters in die des Mehrwert-Ausbeuters gewechselt. Aggressive Verkaufsstrategien und steigende Abonnementpreise bei gleichzeitig sinkenden Qualitäts- und Sorgfaltsstandards machten die Verlagstätigkeit zum profitabelsten Geschäft der Welt, mit Gewinnmargen bis zu 40 \%.8 Diese Symptome prägen bis heute den Kampf der Open-Access-Verfechter gegen die betreffenden Grossverlage. Ein etwas anderes Bild jedoch bieten Rechtsverlage in Deutschland:9 Die wissenschaftlich renommierten sind noch immer in Familienhand; Autoren erhalten für ihre Texte noch immer Honorar, statt Aufforderungen zur unentgeltlichen Peer Review; redaktionelle und handwerkliche Standards sind längst nicht auf dem Niveau der von englischsprachigen Verlagen mitunter komplett nach Indien ausgelagerten Massenproduktion. Dafür sind Preis-, Umsatz- und Gewinnentwicklungen der deutschen Rechtsverlage völlig intransparent und wurden nie systematisch untersucht. Wie sich Open-AccessModelle in dieser undurchschaubar komplexen Landschaft entwickeln lassen, und ob etablierte Verlage sich als Dienstleister verstehen möchten, die ihr Geschäftsmodell künftig auf abgrenzbare Mehrwertleistungen ausrichten statt auf die überkommene (zeitlich und örtlich unbeschränkte) Rechtsausübung, sind wohl Schlüsselfragen für die Zukunft des Open Access in der deutschsprachigen Rechtswissenschaft.

\section{Zukunftsperspektiven und Weiter- führung}

26 In seinem Schlusswort lobte Dirk Verdicchio den vielversprechenden «Einstieg in die Diskussion», erklärte aber auch, dass eine Wiederholung der Tagung einstweilen nicht geplant sei. Erst im Rahmen der anschliessenden Abendgestaltung zeichnete sich ab, dass die Teilnehmer sowohl über das gelungene Konzept der Tagung als auch die Sinnhaftigkeit eines weiteren Austausches einig waren. Eine Folgetagung wurde deshalb für 2018 verabredet, wobei auch deutsche und österreichische Interessenten vermehrt zur Teilnahme ermutigt werden sollen. $\mathrm{Zu}$ diesem Zweck wurde ein Verteiler eingerichtet, der über weitere Schritte bis zur nächsten Open-Access-Tagung informiert; Interessierte können sich dafür mit einer kurzen Mail an openaccess@coll.mpg.de anmelden.

\footnotetext{
van Noorden, Nature 2013, 426.

Vgl. Hamann, JuS 3/2015, 32.
} 